\title{
SOCIOECONOMIC CHARACTERISTICS OF SPEEDING BEHAVIOR
}

\author{
Kyungwoo Kang \\ Department of Transportation Engineering \\ Hanyang University \\ Ansan, Korea. \\ E-mail: kyungwoo@hanyang.ac.kr
}

\begin{abstract}
Summary: Many studies on drivers' speeding behavior have been reported in the last decade. Most of the previous studies, however, have concentrated on the relationship between drivers' speeding behavior and road/vehicle characteristics, without considering other important factors such as personal characteristics and drivers' perception of the speed limit. This paper analyzes Korean drivers' speeding behavior by taking into account such factors as trip characteristics in addition to personal, vehicular, and attitudinal factors. Speeding behavior is measured by a categorical measure over the speed limit, and an ordered probit model is used to econometrically estimate the speeding behavior equation. Results indicated that i) male drivers with higher income tend to drive faster, and experienced drivers drive at higher speeds than others ii) vehicles with more horsepower and vehicles with safety features go slower than vehicles with less safety features iii) trip distance and frequent use of the road are important factors for speed selection behavior, iv) perceived speed limit of the road and expectation of being caught for speeding are important factors for driving behavior.
\end{abstract}

\section{INTRODUCTION}

Recent studies by Jorgensen (1983, 1991), Jorgensen and Polak (1993), Wasielewski (1984) and Rienstra and Rietveld (1996) include detailed variables for speed selection such as attitude for speed selection in analyzing driver behavior. Their studies are limited for practical use, however, because they use only limited variables for driver characteristics. They ignore vehicular characteristics such as safety features of the vehicles. In general, speeding behavior is determined by the combination of physical characteristics of the road, vehicle, and traffic conditions as well as trip purpose. For a given set of road and traffic conditions, speed selection behavior should be determined by the characteristics of the vehicles and drivers, including trip purposes. Actually, we can easily infer the importance of those factors from different speed distributions on the same road segments at the same time. In order to analyze speed selection behavior comprehensively, we should, therefore, include not only traditional physical conditions of the road but also vehicle and drivers' characteristics.

Needless to say, speeding is one of the most critical factors in traffic accidents around the world. This is well known, even though the recent experiences of speed limit increases in the USA show some significant reductions in traffic accidents. Traffic accidents represent one of the most serious economic and social problems in modern society. Also, traffic accidents cause massive traffic congestion in urban areas. Therefore, various factors such as physical characteristics of roads and drivers' behavior should be investigated in order to set optimal speed limits. The main purpose of this research is to study drivers' speeding behavior in Korea using various combinations of variables including vehicles, characteristics of the roads, and drivers' and trip 
purposes. Target groups are restricted to automobile drivers during off-peak hours because the morning and afternoon peak hours in urban areas have severe traffic congestion during which a driver's desired speed cannot be achieved.

This paper has five sections. The first and second sections introduce speed selection behavior and literature review of speed selection models. Section three discusses our survey methodology and data collection method. Section four summarizes the estimation results of the ordered probit analysis for speeding behavior and provides some interesting findings. Finally, concluding remarks and some suggestions for further studies follow.

\section{SPEED SELECTION MODEL}

After a careful review of the existing literature, we have decided that an economic model for speed selection behavior suggested by Jorgensen and Polak (1995) is suitable for our purpose. In their model, drivers are subjectively rational, risk neutral and utility maximizing agents. We also assume that a driver's speed selection is determined by trip time, safety consideration, expected speed limit, and speeding ticket costs. Under these assumptions, the drivers choose their speeds to minimize total travel costs, taking into consideration the existing conditions described above.

To investigate the effect of speed selection behavior in Korea, ordered probit models were estimated. This model was selected because of its ability to analyze ordered categorical response data, that is, the response to select speed to maximize net benefits which is defined as expected benefits minus expected related costs for given speed. The ordered logit model was also considered for estimation. However, in practice the two formulations give very similar results. Therefore the ordered probit model, where the error term is normally distributed, was selected.

\section{DATA COLLECTION METHOD}

\section{Data Collection}

For our model, we need two types of data sets; namely, speed data measured in the survey segment of the road and the characteristics of drivers, roads, vehicles, and trip purposes.

Speed can be measured by using video cameras and detectors. In order to obtain personal data for drivers, however, drivers must be stopped and interviewed in the middle of the road. In some other studies, police assistance is required in order to conduct field interviews and run the license-matching program. In our study, however, we have ruled out police assistance, because a driver's speed selection behavior can be modified significantly when police appear. At the same time, the license-matching method cannot describe the exact driver and passenger because vehicle registrations only show the owner of the vehicle not actual drivers.

In order to avoid such difficulties, we have selected four lanes of each direction of a $1.2 \mathrm{Km}$ restricted access road section in the city of Ansan. Ansan has a population of approximately half million people and is one of the first planned major industrial cities in Korea. In the surveyed section of the road, two speed limit (70Km/hour) traffic signs were installed. During the survey period, no police were present or visible to maintain the speed limit. 
The field survey consists of 18 questions, among which four questions about license number, sex, occupancy, and seat belts are recorded by the interviewers. The remaining 14 questions regarding the characteristics of drivers, vehicles and trip purposes are collected from the interviews with the drivers. The survey was conducted from June to August in 1997 where there were four distinct characteristics of the roads. The weather for all of the days was sunny. Survey time was selected to be 10:30 AM to 5:00 PM, in order to collect various trip purposes. A total of 2,889 vehicle speed observations and 769 field interviews were collected. After a careful review of the vehicle speed data and field data, 581 observations were selected for our analysis in speed selection behavior

\section{ESTIMATION RESULTS}

The model specifications explored the effects of the following various factors on speed selection behavior of Korean drivers:

\section{Personal characteristic effects on speed selection behavior Vehicle and safety characteristics of speed \\ Trip characteristics \\ Perceived likelihood for speeding}

\section{Personal characteristics of speed}

In our sample, male drivers account for about $90 \%$. In order to make sure that our sample represents the national average, we compared our values with national averages. Nationwide, female drivers account for almost 23\% of all drivers, which are much higher than the $10 \%$ of female drivers in our sample. Since the number of driver licenses owned be female are generally much greater than the number of actual drivers on the road, it is reasonable to assume that the sex distribution in our sample does not significantly deviate from the national average.

Other things being equal, male drivers drive faster than female drivers. This result implies that female drivers generally show greater responsibility for the care and welfare of other family members and/or passengers than male drivers do. In terms of driving experiences, drivers with 10 or more years of driving experience drive faster than those of any other category. This behavior is also confirmed by marginal effects such as 0.0675 and 0.2315 , which is clearly higher than 0.0455 and 0.1516, respectively. Regarding educational level, drivers with higher education tends to drive more carefully than drivers with less education, which is confirmed by marginal effects.

\section{Vehicle and safety characteristics of speed}

Among the four variables, vehicle horsepower and an index (airbag, ABS, power steering and automatic transmission are indexed according to their existence so that the index ranges from 0 to 4) of safety features are statistically significant for speed. Expectedly vehicles with greater horsepower tend to travel faster. Also, other things being equal, the fewer the safety features, the higher the speed. This unexpected speeding behavior can be explained by: the higher the value of human life, the more the safety features and vehicles with more horsepower. Obviously, the vehicle with more horsepower has more safety features such as ABS and airbag. These results 
are quite important policy implication indicating that the less safe vehicles tend to increase speeding behavior, which may cause more severe traffic accidents.

\section{Trip characteristics}

Regarding the trip characteristic variables, trip distance is a statistically significant factor for speeding behavior. The longer the trip distances the higher the speed. Even though statistically not significant, trip frequency of the road indicated that drivers who were more familiar to the road characteristics tended to drive more carefully than drivers with less knowledge of the road. This trend also increases the probability of traffic accidents.

\section{Perceived likelihood for speeding}

Among the perceived aspects of speeding such as likelihood of being caught for speeding, expected financial loss for speeding, and perceived speed limit for the section of road, perceived speed limit of the road and likelihood of being caught for speeding are statistically significant factors for speed selection behavior. Ironically, drivers who speed have higher expectations of being caught. Despite that, drivers who speed already know that he/she is violating the speeding law, but he/she does not reduce speed, which is another factor for higher accident risks.

\section{CONCLUDING REMARKS}

Unfortunately, Korea has one of the highest traffic accident rates among the OECD countries. Korea recorded over 10,000 deaths each year from traffic accidents, which is 3-5 times higher than the USA and Japan in terms of vehicle registrations. And speeding is the major factor for traffic accidents. In this study, we have estimated speeding behavior by using data collected for speed and 18 different variables including personal characteristics of drivers, vehicle and safety information, trip purpose, and perceived information about speeding in Korea.

We have estimated an ordered probit model in order to capture different impacts on speed selection behavior. Results indicated that i) male drivers with higher income tend to drive faster, and experienced drivers drive at higher speeds than others ii) vehicles with more horsepower and vehicles with safety features go slower than vehicles with less safety features iii) trip distance and frequent use of the road are important factors for speed selection behavior, iv) perceived speed limit of the road and expectation of being caught for speeding are important factors for driving behavior.

The result of this research has revealed that speed selection behavior is a combination of different factors of personal, vehicle, safety, trip and drivers' perception. Some factors such as personal characteristics of drivers are not controllable for transportation policy purposes.

Factors such as perceived impacts on drivers are certainly controllable, however, by using appropriate transportation policies. Especially, increases in the awareness of the speed limit and perceived financial loss of accidents are very important factors, which discourage speeding. Drivers with more knowledge of the speed limit tend to observe the posted speed limit more strictly. In order to reduce speeding and accidents in urban areas, more safety related traffic signs such as speed limit signs, fines for speeding, and police presence on the road could be useful instruments. 
Finally, as shown from the international comparisons, speed selection behavior is quite different from one country to another. Further studies are, therefore, needed for more conclusive evidence in speed selection behavior before introducing of new measures for speeding policy such as an increase in the speed limit.

\section{ACKNOWLEDGEMENTS}

The financial support by the Advanced Highway Research Center of Hanyang University is gratefully acknowledged.

\section{References}

Jorgensen F.J. (1991) Drivers' Speed Selection and Traffic Safety: In Norwegian, Nordland College, Bod.

Jorgensen, F.J. (1983) Measuring Car Drivers' Skill - An Economist's View, Accident Analysis and Prevention 25.2, 555-559.

Jorgensen F.J. and Polak J. (1993) The Effect of Personal Characteristics on Drivers' Speed Selection: An Economic Approach, Journal of Transport Economics and Policy 27.3, 237-252.

Kanellaidis G., Golias, J. and Zarifopoulos, K. (1995) A Survey of Drivers' Attitudes Toward Speed Limits Violations, Journal of Safety Research 26.1, 31-40.

Leonard E. and Wasielewski, P. (1983) Risky Driving Related to Driver and Vehicle Characteristics, Accident Analysis and Prevention 15. 2, 121-136.

Leonard E. and Wasielewski, P. (1982) Do Accident-Involved Drivers Exhibit Riskier Everyday Driving Behavior, Accident Analysis and Prevention 14.1, 57-64, (1982).

Mannering F.L., Abu-Eisieh, S.A. and Arnadottir, A.T. (1990) Dynamic Traffic Equilibrium with Discrete/Continuous Econometrics Models, Transportation Science 24.2, 105-116.

McFarland W.F. and Chui M. (1987) The Value of Travel Time: New Elements Developed Using a Speed Choice Model. Transportation Research Record 1116, TRB, National Research Council, Washington, D.C.15-21.

Mohring H. (1965) Urban Highway Investments. In Measuring Benefits of Government Investments.

Preusser D.F., Williams A.F. and Lund A.K. (1991) Characteristics of Belted and Unbelted Drivers, Accident Analysis and Prevention 23. 6, 472-482.

Ranney T.A. (1994) Models of Driving Behavior: A Review of Their Evolution, Accident Analysis and Prevention 26. 6, 733-750.

Rienstra S.A. and Rietveld, P. (1996) Speed Behavior of Car Drivers: A Statistical Analysis of acceptance of changes in Speed Polices in the Netherlands, Transportation Research - D, 1. 2, 97-110.

Wasielewski P. (1984) Speed as a Measure of Driver Risk: Observed Speed versus Driver and Vehicle Characteristics. Accident Analysis and Prevention 16. 2, 89-103. 